\title{
IRVAN syndrome
}

INSERM

\section{Source}

INSERM. (1999). Orphanet: an online rare disease and orphan drug data base. IRVAN syndrome. ORPHA:209943

A rare retinal vasculopathy disease characterized by idiopathic retinal vasculitis (IRV), aneurysmal dilations (A) at arteriolar bifurcations, and neuroretinitis $(N)$, which if untreated progresses to peripheral capillary non-perfusion, retinal neovascularization, and macular exudation, leading to severe, bilateral vision loss. 